\title{
Development of large area resistive electrodes for ATLAS NSW Micromegas
}

\author{
Atsuhiko Ochi ${ }^{1, a}$, on behalf of the ATLAS Muon Collaboration \\ ${ }^{1}$ Kobe University, Kobe 657-8501, Japan
}

\begin{abstract}
Micromegas with resistive anodes will be used for the NSW upgrades of the ATLAS experiment at LHC. Resistive electrodes are used in MPGD devices to prevent sparks in high-rate operation. Large-area resistive electrodes for Micromegas have been developed using two different technologies: screen printing and carbon sputtering. The maximum resistive foil size is $45 \times 220 \mathrm{~cm}$ with a printed pattern of $425-\mu \mathrm{m}$ pitch strips. These technologies are also suitable for mass production. Prototypes of a production model series have been successfully produced. In this paper, we report the development, the production status, and the test results of resistive Micromegas.
\end{abstract}

\section{Requirements for ATLAS NSW Micromegas and resistive strips}

The ATLAS detector will be upgraded in view of the luminosity increase of LHC. For endcap muon systems, a New Small Wheel (NSW) will be introduced instead of the current inner endcap muon detectors. A NSW consists of two types of gaseous detectors: sTGC and Micromegas. In this NSW, Micromegas are mainly designed for high resolution tracking, and to provide auxiliary trigger signals [1]. The NSW covers $1.3<\eta<2.7$ ( $\eta$ is pseudo rapidity) and will be placed $7 \mathrm{~m}$ from the interaction point. NSW installation is planned for the next LHC long shutdown (2019-2020).

For increased luminosity, we require an endcap muon detector with both high position resolution and tolerance to a high rate of Highly Ionizing Particles (HIP). The position resolution requirements for the $\eta$ direction are less than $100 \mu \mathrm{m}$, and they are a few mm for the $\phi$ direction (azimuth angle around the beamline). At the NSW, the neutron and $\gamma$ ray irradiation rate will rise up to $15 \mathrm{kHz} / \mathrm{cm}^{2}$. Such incident particles might cause sparks in the detector because the deposit energy inside it could exceed the Raether limit [2-4]. To stably operate the muon detector to meet such requirements, ATLAS Micromegas is designed with resistive strips as anode electrodes [1].

The schematic structure of the NSW Micromegas is shown in Fig. 1. The avalanche charges on the resistive strips induce a signal on the readout strips. The readout strips are made from Printed Circuit Boards (PCBs). Resistive strips are formed on thin $(50 \mu \mathrm{m})$ polyimide foils and attached to the readout PCBs.

A NSW consists of 16 sectors (eight large and eight small types), as shown in Fig. 2. The dimensions of the small and large sectors are shown in Fig. 3. The sectors consist of two modules, which have $3+5$ readout boards. The shape of the resistive strip foils is almost identical as

\footnotetext{
ae-mail: ochi@kobe-u.ac.jp
}

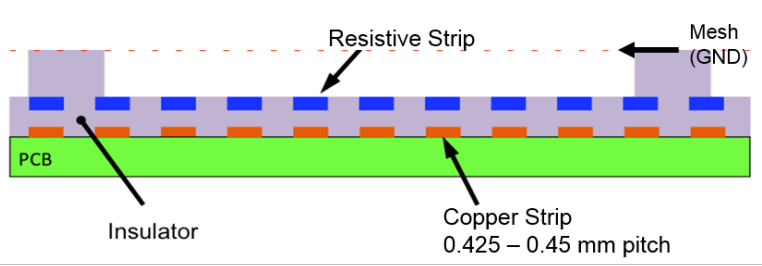

Figure 1. Schematic structure of Micromegas with resistive anodes designed for ATLAS NSW

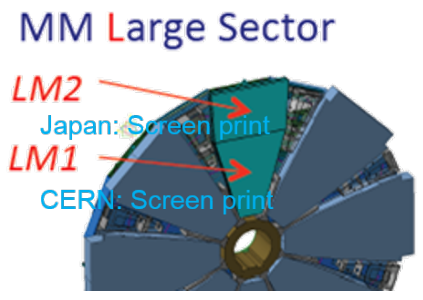
MM Small Sector

Figure 2. ATLAS NSW consists of eight large and eight small sectors. There are two Micromegas modules for each sector: LM1 and LM2 (large sectors) and SM1 and SM2 (small sectors). Resistive foils for module-0 for LM1 are made by screen printing in CERN, those for LM2 and SM1 are made by screen printing in Japan, and those for SM2 are made by carbon sputtering in Japan.

the readout PCBs. The maximum foil size is $45 \times 222 \mathrm{~cm}$ with a printed pattern of $425-450 \mu$ m pitch strips. An example of a magnified resistive strip pattern is shown in Fig. 4. The resistivity requirements of the anode strip are $20 \mathrm{M} \Omega / \mathrm{cm}$.

Before full production of the NSW Micromegas modules, test chambers are produced for checking the technical availability. Those prototype modules are called Module0 s [5]. All of the chamber types in the large and small 


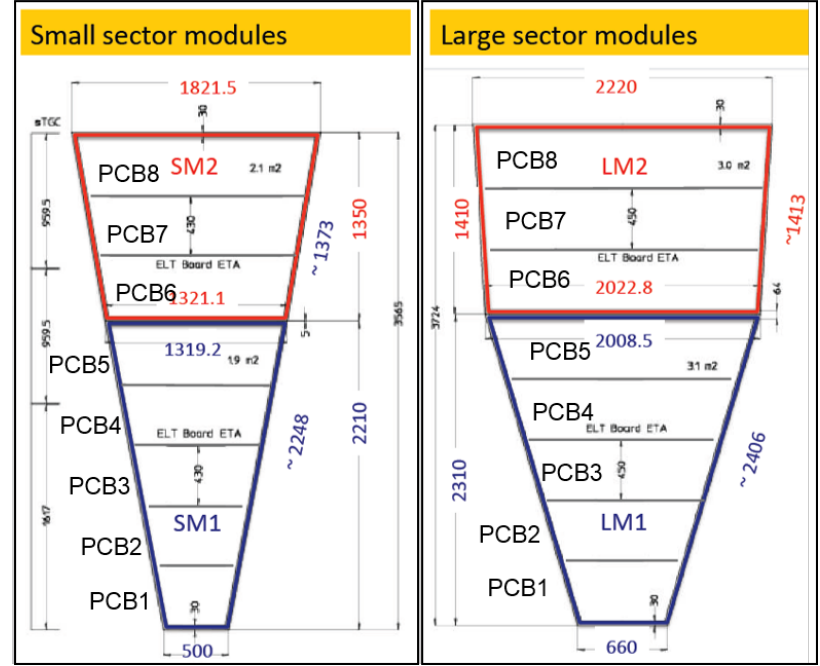

Figure 3. Size parameters of Micromegas modules for both small and large sectors

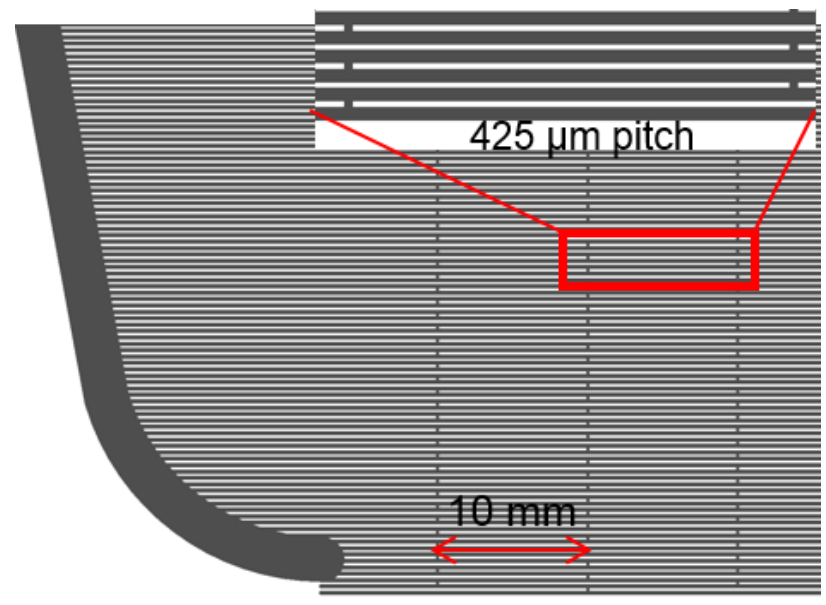

Figure 4. Example of resistive strip pattern. The thick line on left is HV supplier line. The pitch of the strips is $425 \mu \mathrm{m}$ with interconnecting lines with $1-\mathrm{cm}$ spacing between strips.

sectors were produced for the Module-0 prototype. There are 32 types of resistive strip patterns.

Two different types of resistive strips foils have been developed for NSW Micromegas. One is carbon sputtering with a liftoff method, and the other is the screen printing of carbon paste. These two production processes have been studied for large-size resistive strip foils in module-0 production. The foils for the SM2 type (Fig. 3) were made using carbon sputtering, and those for the SM1/LM2 types were made using screen printing.

\section{Carbon sputtering and liftoff}

Carbon sputtering is an excellent method for making resistive electrodes for MPGDs [6]. The fine structure of electrodes can be formed by the liftoff method (Fig. 5). In the first step, (a) the photoresist on the polyimide sub-

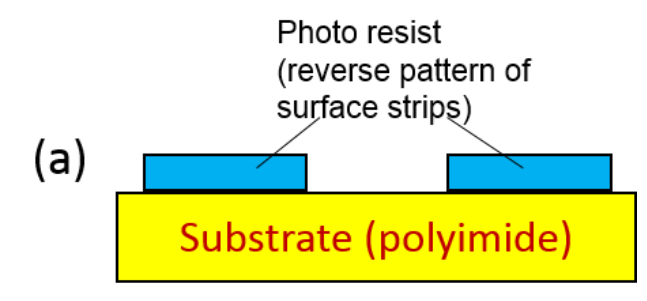

(b)

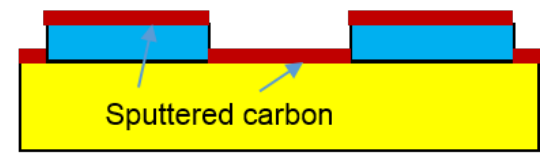

(c)

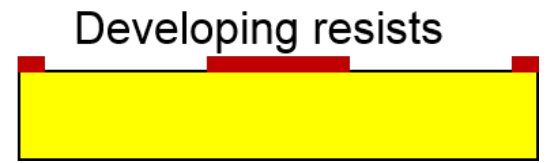

Figure 5. Resistive patterning process using carbon sputtering and liftoff process: (a) photoresist on polyimide substrate is etched by photolithography; (b) carbon is sputtered on surface; (c) remaining resist is removed by strongly alkaline water. The alkaline attacks to only photo-resists from the side of them.

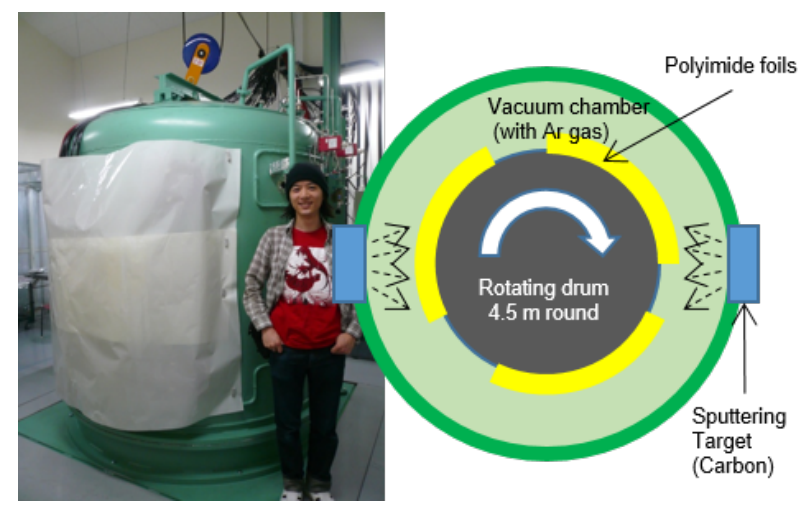

Figure 6. Large-size sputter chamber (Be-Sputter Co., Ltd.) and a schematic diagram of sputtering machine

strate is etched by photolithography. Second, (b) carbon is sputtered on the surface. Finally, (c) the remaining resist is removed by strongly alkaline water. Making large size patterns using the photolithography is a common technology for producing PCBs. Large-size $(1 \times 4.5 \mathrm{~m}$ maximum, see Fig. 6) sputtering equipment is also available from Be-Sputter Co., Ltd. Resistive pattern masking and liftoff process were made by Raytech Inc. The sputtered carbon's resistivity can be controlled by its thickness and nitrogen doping, which has been newly developed in our studies. The target resistivity of NSW resistive foils is about $0.8 \mathrm{M} \Omega / \mathrm{sq}$., which corresponds to the strip resistivity of $20 \mathrm{M} \Omega / \mathrm{cm}$ with $0.4 \mathrm{~mm}$ pitch. This value is easily available for thin (70-nm thick) carbon thickness with appropriate nitrogen doping [6]. 


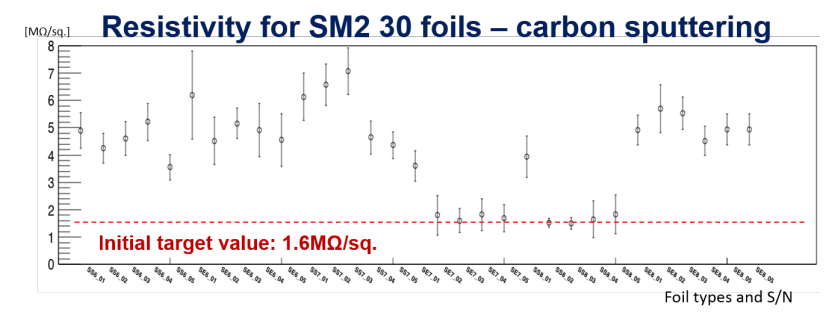

Figure 7. Surface resistivity for 30 resistive foils produced using carbon sputtering for SM2 type modules
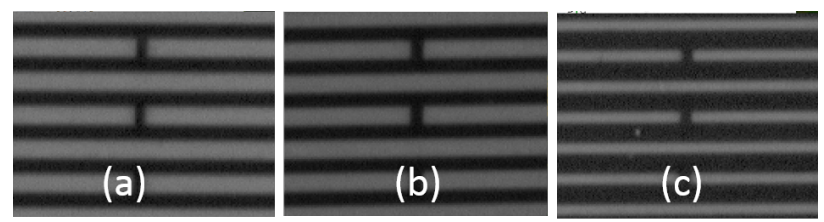

Figure 8. Microscopic image of sputtered electrodes. Differences in line width can be seen for different foils.

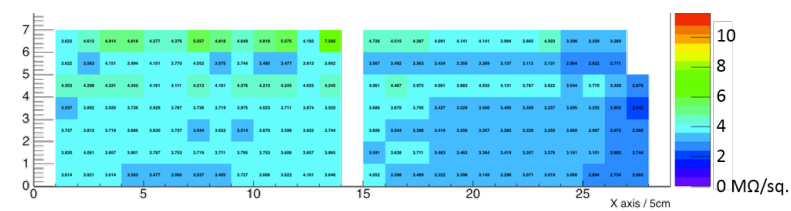

Figure 9. Surface resistivity map of one sputtered foil. Colors correspond to surface resistivity for each $5 \times 5 \mathrm{~cm}$ area.

For the module- 0 prototype, we produced 30 foils using the carbon sputtering method. In the first large area production, we found some large variabilities of the resistivity (Fig. 7), caused by the differences of the width of the resistive strips due to mistakes in the initial mask pattern (Fig. 8). But the uniformity of the resistivity within each foil is very good (less than $50 \%$ resistivity fluctuations as shown in Fig. 9).

\section{Screen printing}

The resistive strip patterns for NSW Micromegas can also be printed on substrate by a screen printing technique. The carbon loaded epoxy, RS12115 (from ESL ElectroScience), was used as a printing paste. The printing process was done by Matsuda-Screen Inc., where a large (2.6 $\times 1.3 \mathrm{~m}$ ) screen printing machine is available in a clean room (Fig. 10). After printing the paste on the substrate, the foils were cured by a large-size oven at $170^{\circ} \mathrm{C}$ for two hours. The quality of the patterns and the resistivity is controlled by the line/space (L/S) width on the screen plate. For printing a $400-\mu \mathrm{m}$ pitch of strip patterns, five L/S patterns were tested: $\mathrm{L} / \mathrm{S}=150 \mu \mathrm{m} / 250 \mu \mathrm{m}, 180 \mu \mathrm{m} / 220 \mu \mathrm{m}$, $200 \mu \mathrm{m} / 200 \mu \mathrm{m}, 220 \mu \mathrm{m} / 180 \mu \mathrm{m}$, and $250 \mu \mathrm{m} / 150 \mu \mathrm{m}$. Fig. 11 shows microscopic pictures of the printed patterns and a resistivity table for each pattern. From this test, we conclude that a $150-\mu \mathrm{m}$ line width was too narrow to make

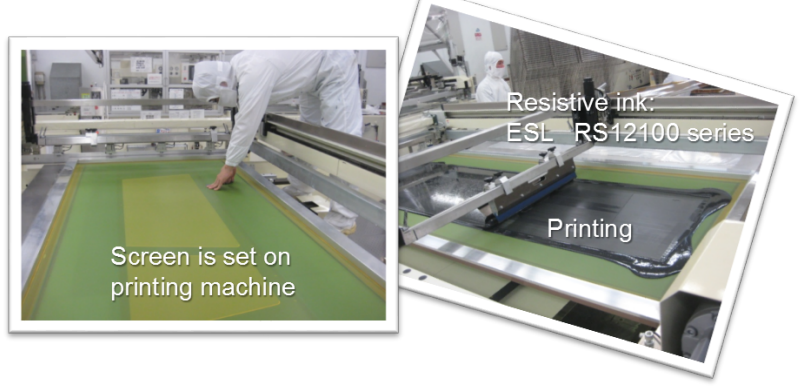

Figure 10. Pictures of screen printing for resistive foils (Matsuda-Screen Inc.). Large printing machine was used in a clean room.

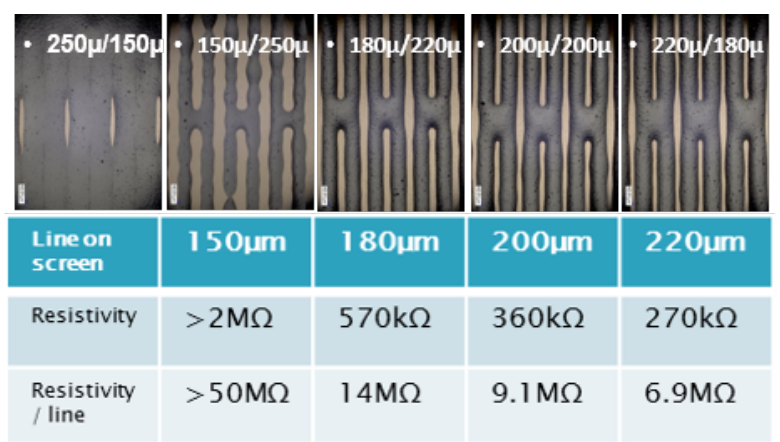

Figure 11. Microscopic pictures of screen-printed resistive strips and measured resistivities

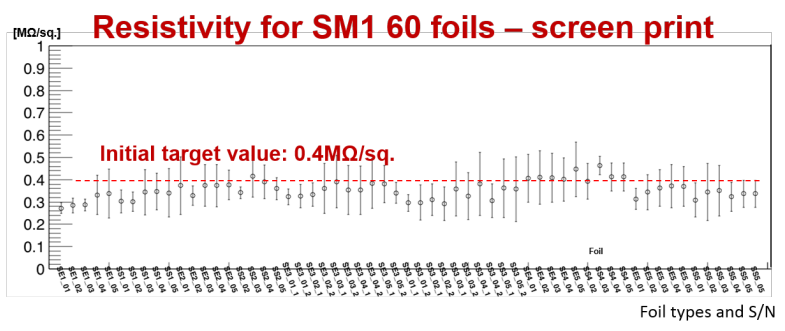

Figure 12. Surface resistivity for 60 resistive foils produced using screen printing. Those foils were made for SM1 type module.

strips, and a $150-\mu \mathrm{m}$ space width was too narrow to separate each strip. We chose $\mathrm{L} / \mathrm{S}$ values of $180 \mu \mathrm{m}$ and 220 $\mu \mathrm{m}$, which show the closest resistivity of $20 \mathrm{M} \Omega / \mathrm{cm}$.

For the module- 0 prototype, we produced 60 foils using the screen-printing method. Based on our previous experiment, the resistive value will double after the foils are attached to the readout PCB board. Then the initial target resistivity was set to $0.4 \mathrm{M} \Omega / \mathrm{sq}$. Fig. 12 shows the results of the resistivity measurements for each screenprinted foil. The resistivity of all the foils satisfies our requirement. 


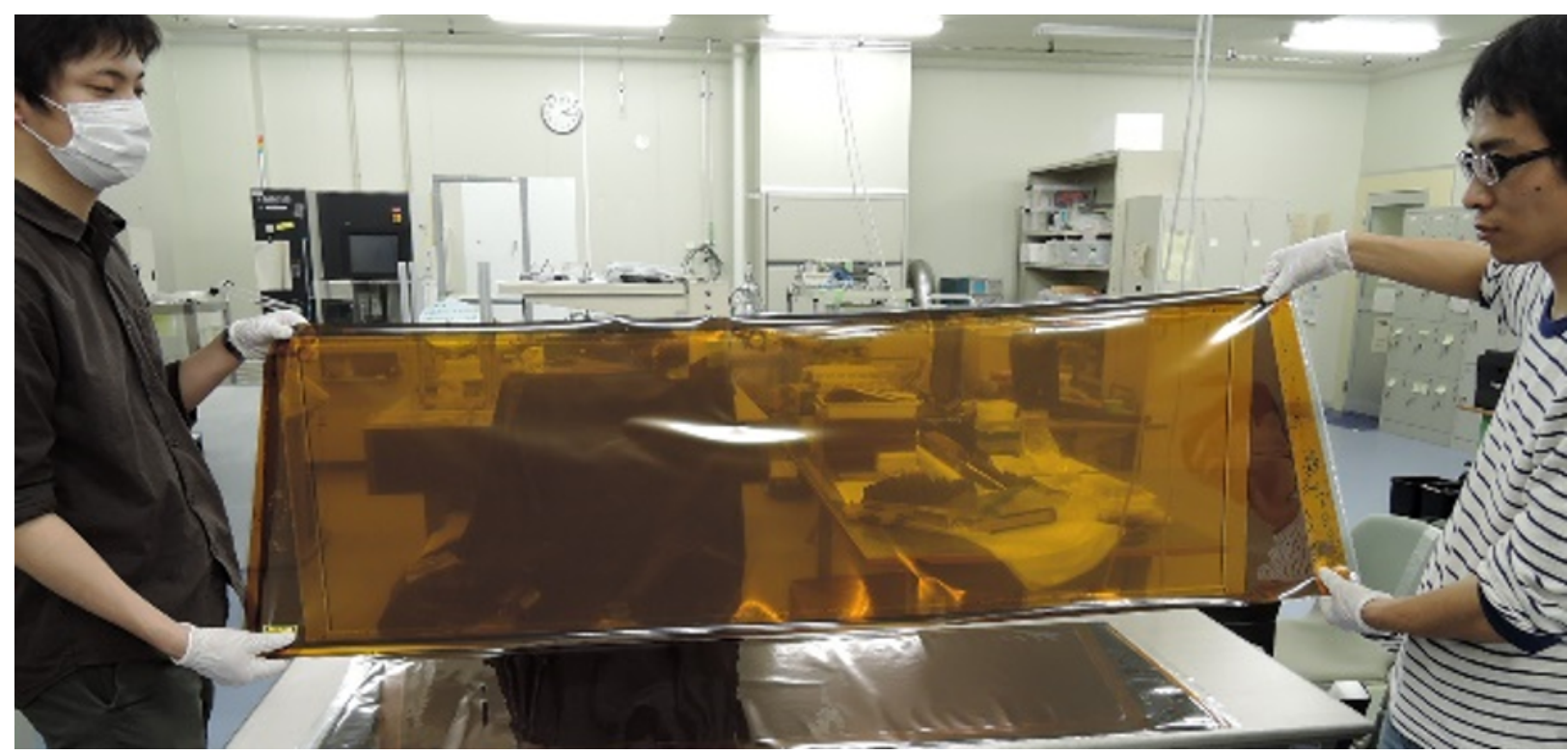

Figure 13. Successfully produced module- 0 foils. This picture is one large foil made by sputtering method.

\section{Conclusion: Comparing the two technologies}

We used both carbon sputtering and screen printing to produce Micromegas resistive foils for module-0s. Fig. 13 shows a large resistive foil for the NSW module- 0 . The pattern precision of carbon sputtering greatly surpasses that of screen printing (Figs. 8 and 11). However, the availabilities and qualities of both are adequate for NSW series production. From the viewpoint of production cost, screen printing is suitable for mass production, and we need almost 2000 foils for NSW construction. A rough cost estimation suggests that full production cost using screen printing is about three times lower than carbon sputtering. Therefore, we chose screen printing technology as a first candidate for resistive foil production technology for the ATLAS NSW Micromegas.
This work was supported by JSPS KAKENHI Grant Numbers 23340072, 26104707, and 15H02092.

\section{References}

[1] ATLAS Collaboration, "New Small Wheel Technical Design Report", CERN-LHCC-2013-006 (2013)

[2] H. Raether, Electron Avalanches and Breakdown in Gases. London, UK: Butterworth, (1964)

[3] A. Bressan, et al., Nucl. Instr. Meth.A 424 (1999) 321

[4] S. Bachmann, et al., Nucl. Instr. Meth.A 479 (2002) 294

[5] J. Bortfeldt et al., in this volume of proceedings.

[6] A. Ochi et al., Proc. of Sci. (TIPP2014) 35 